\title{
FRECUENCIA DE DNA-HPV-H EN CÉLULAS ESCAMOSAS ATÍPICAS SERIE DE CASOS: ASC-US y LSIL
}

Miryam B. Puerto de Amaya*, Mercedes Olaya Contreras MD**, Karen Rey Cediel***, Katherine Diaz Jiménez***, Carlos H. Pérez M.MD****

\section{Resumen}

Objetivo: determinar mediante reacción en cadena de la polimerasa (PCR) la infección por el virus del papiloma humano de alto riesgo (DNA-HPV-H) y la presencia de cambio morfológico con atipia en la citología de mujeres que laboran en un hospital y un ente educativo. Métodos: serie de casos de muestras cérvico uterinas con citologías convencional y en base líquida, y PCR para DNA-HPV-H; se incluyeron las pacientes que tuvieron uno o más resultados positivos en citología (ASC-US y LSIL). Resultados: la tipificación de DNA-HPV-H fue positiva en 12 de 41 casos. Se observó un mayor número de citologías con atipia celular en el rango de mujeres jóvenes (22-49 años), comparado con las mayores (50-72 años). Los casos positivos para DNA-HPV-H tanto en citología convencional como en base líquida fue similar. Hubo 11 con infección simple y uno múltiple positivo para alto riesgo (HPV-H y HPV-16). Conclusiones: la PCR identificó 12 pacientes infectadas con HPV de alto riesgo, 11 con infección simple y una múltiple; el grupo que predominó fue el HPV-H (9 casos), seguido del subtipo HPV-18 (5) y por HPV-16 (1).

Abreviaturas: ASC-US atipia indeterminada de células escamosas; ASC-H, atipia que no descarta alto grado; HPV, virus del papiloma humano; LSIL, lesión escamosa intraepitelial de bajo grado; HSIL, lesión escamosa intraepitelial de alto grado.

Palabras clave: virus del papiloma humano, cáncer de cuello uterino, citología, neoplasia cervical intraepitelial.

\section{DNA-HPV-H DETECTION IN ATYPICAL SQUAMOUS CELLS. A CASE SERIES: ASC-US AND LSIL}

\section{Abstract}

Objective: to determine high-risk human papillomavirus (DNA-HPV-H) infection and atypical changes by the polymerase chain reaction (PCR) method based on cytology among women who work at a hospital and a school. Methods: case series based on conventional or liquid-based cytology of cervical samples and PCR testing for DNAHPV-H; patients with one or more positive results in their cytology (ASC-US and LSIL) were included. Results:

Fecha recibido: septiembre 20 de 2013 Fecha aceptado: diciembre 20 de 2013

* Bacterióloga y Citohistóloga. Docente de patólogos y citólogos desde 1967. Profesora. Asistente, Fundación Universitaria de Ciencias de la Salud. Bogotá DC, Colombia.

** Médica patóloga perinatal. Profesora Asistente. Pontificia Universidad Javeriana. Hospital Universitario San Ignacio. Bogotá DC, Colombia.
*** Estudiantes de citohistotecnología. Fundación Universitaria de Ciencias de la Salud. Bogotá DC, Colombia.

**** Médico ginecólogo. Colposcopista, Profesor Titular. Fundación Universitaria de Ciencias de la Salud. 
DNA-HPV-H typing was positive in 12 to 41 cases. Atypical cells were mostly present in younger women (22-49 years), compared with older women (50-72). The number of DNA-HPV-H positive samples was similar for liquidbased and conventional cytology. Eleven cases had a simple infection and one a multiple positive result for highrisk (HPV-H and HPV-16). Conclusions: high-risk HPV infection was identified by PCR in 12 patients, 11 had a simple infection and one a multiple infection; HPV-H positive ( 9 cases) samples predominated, followed by subtype HPV-18 (5) and by HPV-16 (1) type.

Key words: human papillomavirus, cervical cancer, cytology, cervical intraepithelial neoplasia

\section{Introducción}

La evolución de las costumbres ha llevado a la sociedad moderna a frecuentes infecciones virales, las cuales pueden desencadenar procesos inflamatorios y precancerosos, siendo más frecuentes en mujeres jóvenes. ${ }^{1}$ Las causadas por tipos oncogénicos (alto riesgo) de HPV han sido reconocidas como el evento indispensable en el desarrollo del cáncer cervical y sus lesiones precursoras. ${ }^{2,3}$ La persistencia de estas infecciones (la mayoría de transmisión sexual $^{4,5}$ ) en el cuello uterino ${ }^{6}$, llevan a un espectro de cambios morfológicos celulares con atipia de diferente intensidad clasificados según el sistema Bethesda ${ }^{7,8}$ en: ASC-US, ASC-H, LSIL, HSIL y carcinoma invasivo.

Paralelo al cambio de costumbres, la ciencia y la tecnología han desarrollado pruebas auxiliares como herramientas más sensibles y reproducibles para detectar el riesgo de la infección y su persistencia, permitiendo identificar los diferentes subtipos del HPV, que en conjunto con la citología convencional o la de la base líquida, ayudan a clarificar el cambio celular ${ }^{9}$ en mejora de una exactitud diagnóstica, de una remisión y de un tratamiento adecuado y oportuno. ${ }^{10}$

Para este estudio, se utilizó citología convencional y en base líquida para identificar a mujeres con resultados clasificados como ASC-US o LSIL a quienes después se les realizó pruebas de PCR (reacción en cadena de la polimerasa COBAS ${ }^{\circledR} 4800$ ).

\section{Materiales y métodos}

Población: estudio de serie de casos, se tomaron muestras a 121 mujeres que participaron en la $11^{\mathrm{a}} \mathrm{y}$ $12^{\text {a }}$ Jornadas de Salud Ocupacional en un hospital de cuarto nivel, en Bogotá DC (Colombia), del 20 al 22 de septiembre de 2011 y 25 al 29 de junio de 2012 . Se les explicó la finalidad del estudio y aceptaron firmar el consentimiento informado para participar en la investigación. De este grupo, se incluyeron quienes tuvieran una o más pruebas positivas para citología convencional o en base líquida. Se excluyeron las que no firmaron el consentimiento informado o fueran histerectomizadas. Para la recolección de datos fue utilizado el instrumento de anamnesis y resultados en citología, el análisis se basó en la clasificación de Bethesda 2001. Se midió: edad, número de gestaciones, método de planificación y hábito de fumar.

Muestra citológica: Convencional: el material para el estudio fue obtenido en la consulta de ginecología bajo colposcopio por los especialistas, quienes elaboraron los extendidos convencionales y guardaron los elementos de toma (citocepillo Rovers ${ }^{\circledR}$ ) en un vial, para luego realizar las preparaciones en base líquida y las determinaciones de biología molecular, siendo ésta una muestra compartida. ${ }^{11,12}$

Base líquida: estas muestras se procesaron en el laboratorio de citología de la Facultad de Citohistología, de la FUCS, según el manual del equipo BectonDickinson ${ }^{\circledR}$. Los viales con la muestra residual fueron guardados y refrigerados a $4^{\circ} \mathrm{C}$. 
Prueba DNA-HPV-H: del material residual contenido en el vial de las 41 mujeres, se realizó la prueba molecular con el equipo Cobas ${ }^{\circledR} 4800$ (Laboratorios Roche SA). Para un primer grupo de doce subtipos de alto riesgo (HPV-H: 31, 33, 35, 39, 45, 51, 52 56, $58,59,66$ y 68$)$ y por separado otros dos genotipos considerados de más alto riesgo HPV-16 y HPV-18, siendo el 16 el responsable de cerca del $60 \%$ del cáncer cuello uterino y el HPV 18 representa otro $10 \%{ }^{13,14}$, para un total de 14 subtipos.

Procesamiento y análisis: tanto las citologías convencionales como las de base líquida se colorearon con Papanicolaou y se clasificaron según Bethesda 2001. La primera lectura la realizó una citohistóloga; todos los casos con atipia celular y el 10\% de los negativos fueron revisados por una patóloga. Los formatos de los resultados de citología una vez diligenciados y firmados fueron entregados en la oficina de Coordinación de Salud Ocupacional, para hacerlos llegar a cada una de las participantes y su posterior remisión a la consulta de colposcopia, según las recomendaciones de las guías de manejo para estos casos. ${ }^{15}$

\section{Resultados}

Se analizaron en total 121 muestras, de las cuales 41 cumplieron con los criterios de inclusión, constituyendo el grupo de estudio. Se observó un mayor número de casos con atipia celular en el rango de mujeres jóvenes (22-49 años), comparado con el grupo de las mayores (50-72 años) (Tabla 1).

La determinación de los casos positivos para DNAHPV-H tanto en citología convencional como en base líquida fue similar, presentándose once casos de infección simple (HPV-H nueve, HPV-16 dos y HPV-18 dos) y un caso de infección múltiple positivo para el grupo de alto riesgo (HPV-H y HPV-16) (Tablas 2 y 3). Las variables clínicas frente a HPV-H llamaron

Tabla I. Características clínicas y demográficas según citología convencional y en base líquida

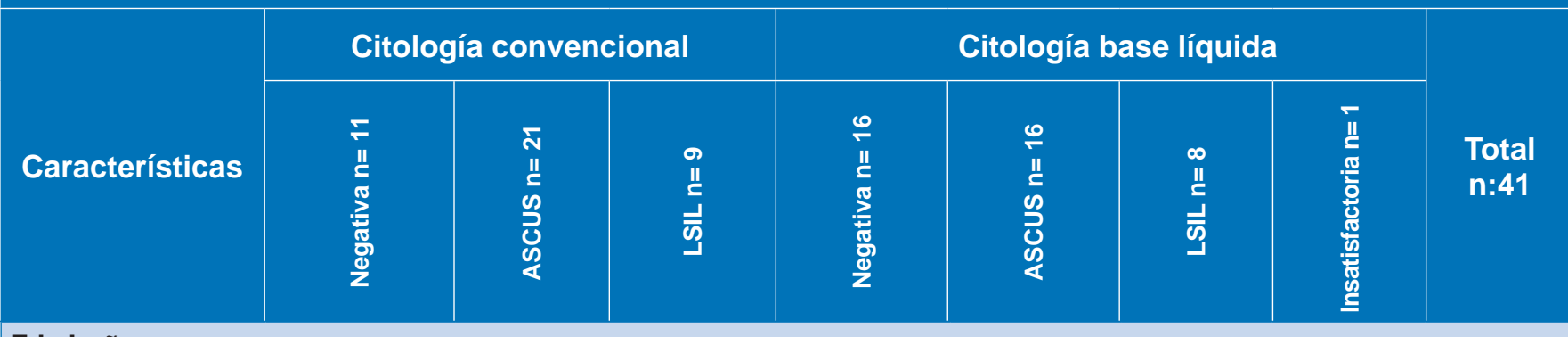

Edad, años

\begin{tabular}{|c|c|c|c|c|c|c|c|c|}
\hline 22 a 49 & 9 & 18 & 7 & 13 & 13 & 6 & 1 & 34 \\
\hline 50 a 72 & 2 & 3 & 2 & 2 & 3 & 2 & 0 & 7 \\
\hline
\end{tabular}

Hábito de fumar, $n$

\begin{tabular}{|l|l|c|c|c|c|c|c|c|}
\hline $\mathrm{Si}$ & $\mathrm{I}$ & 1 & 1 & 0 & 2 & 1 & 0 & 3 \\
\hline No & 9 & 18 & 6 & 14 & 12 & 6 & 1 & 33 \\
\hline No hay reporte & 1 & 2 & 2 & 2 & 2 & 1 & 0 & 5 \\
\hline Gestaciones, $\mathbf{n}$ & & &
\end{tabular}

\begin{tabular}{|c|c|c|c|c|c|c|c|c|}
\hline \multicolumn{9}{|l|}{ Gestaciones, n } \\
\hline 3 a 6 & 2 & 6 & 3 & 3 & 5 & 2 & 1 & 11 \\
\hline 0 a 2 & 7 & 14 & 5 & 12 & 9 & 5 & 0 & 26 \\
\hline No hay reporte & 2 & 1 & 1 & I & 2 & 1 & 0 & 4 \\
\hline \multicolumn{9}{|c|}{ Método de Planificación, n } \\
\hline No & 0 & 6 & 5 & 4 & 3 & 4 & 0 & II \\
\hline $\mathrm{Si}$ & 13 & 7 & 2 & 10 & 8 & 3 & 1 & 22 \\
\hline No hay reporte & 4 & 2 & 2 & 2 & 5 & 1 & 0 & 8 \\
\hline
\end{tabular}


la atención porque entre gestantes de 0 -2 se registraron ocho casos con respecto a gestantes 3-6 tres casos (Tabla 4).

\section{Disc usión}

La oportunidad de realizar este estudio de serie de casos para tamizaje en citología cérvicouterina utilizando conjuntamente las técnicas de citología convencional, la de base líquida y la prueba molecular para HPV oncogénico, permitió establecer los verdaderos casos con atipia (12:41 casos) para ser enviados a la consulta de ginecología, según las recomendaciones y las guías de manejo. ${ }^{16,17} \mathrm{La}$ literatura sugiere el uso de nuevas tecnologías como citología en base líquida y PCR que en conjunto con

\begin{tabular}{|c|c|c|c|c|c|c|}
\hline \multirow{2}{*}{$\begin{array}{l}\text { Citología } \\
\text { convencional }\end{array}$} & \multirow{2}{*}{ n 41} & \multicolumn{5}{|c|}{ Tipificación HPV } \\
\hline & & Negativa & HPV-H & HPV-H 16 & HPV- H 18 & Invalidada \\
\hline Negativa & 11 & 7 & 2 & 1 & 0 & I \\
\hline ASCUS & 21 & 15 & 2 & 0 & I & 3 \\
\hline LSIL & 9 & 3 & 5 & I & I & 2 \\
\hline
\end{tabular}

\begin{tabular}{l|c|c|c|c|c|c|}
\multicolumn{6}{c}{ Tabla 3. Frecuencia de citología en base líquida respecto a tipificación DNA-HPV-H } \\
\hline \multirow{2}{*}{$\begin{array}{c}\text { Citología base } \\
\text { líquida }\end{array}$} & $\mathrm{n} 41$ & Negativa & HPV-H & HPV-H I6 & HPV-H I8 & Invalidada \\
\cline { 2 - 7 } Negativa & 16 & II & I & 0 & I & 3 \\
\hline ASCUS & 16 & II & 3 & I & 0 & 3 \\
\hline LSIL & 8 & 2 & 5 & I & I & 0 \\
\hline Insatisfactoria & I & I & 0 & 0 & 0 & 0 \\
\hline
\end{tabular}

\begin{tabular}{|c|c|c|c|c|c|c|}
\hline \multicolumn{7}{|c|}{ Tabla 4. Características clínicas y demográficas con relación a HPV } \\
\hline Características & \multirow{2}{*}{$\begin{array}{c}\text { HPV negativo } \\
n=29\end{array}$} & \multirow{2}{*}{$\begin{array}{c}\text { HPV positivo } \\
n=12\end{array}$} & \multicolumn{4}{|c|}{ Genotipo de HPV } \\
\hline$n=41$ & & & HPV-H & HPV-H 16 & HPV-H 18 & Invalidada \\
\hline Edad, Mediana (RIQ) & NA & $33(36.6)$ & NA & NA & NA & NA \\
\hline 22 a 49 & 20 & 10 & 8 & 2 & 1 & 4 \\
\hline 50 a 72 & 5 & 2 & 1 & 0 & 1 & 2 \\
\hline \multicolumn{7}{|l|}{ DATOS DEMOGRÁFICOS } \\
\hline \multicolumn{7}{|l|}{ Hábito de fumar } \\
\hline $\mathrm{Si}$ & 1 & 1 & 1 & 0 & 0 & 1 \\
\hline No & 20 & 10 & 7 & 2 & 2 & 5 \\
\hline No hay reporte & 4 & 1 & 1 & 0 & 0 & 0 \\
\hline \multicolumn{7}{|l|}{ Gestaciones } \\
\hline $3-6$ & 7 & 3 & 3 & 0 & 0 & 3 \\
\hline $0-2$ & 15 & 8 & 6 & 2 & 1 & 3 \\
\hline \multicolumn{7}{|l|}{ Métodos de planificación } \\
\hline $\mathrm{Si}$ & 13 & 6 & 4 & 0 & 1 & 3 \\
\hline No & 5 & 6 & 4 & 0 & 2 & 2 \\
\hline \multicolumn{7}{|l|}{ Resultado de citología } \\
\hline Citología convencional & 25 & 12 & 9 & 2 & 2 & 6 \\
\hline Citología en base líquida & 25 & 12 & 9 & 2 & 2 & 6 \\
\hline
\end{tabular}

NA: No Aplica 
la citología convencional contribuyen a disminuir la prevalencia de cáncer de cuello uterino.

ASC-US representan cerca de 5\% de los análisis de citología según el estudio ALTS 2006 y alrededor de $50 \%$ de estos casos son positivos para HPV-H, como lo reporta la publicación ALTS 2003. En esta serie de casos no se tienen porcentajes para comparar con los hallazgos de los estudios publicados; sin embargo se observó que en el grupo de 41 muestras clasificadas con atipia celular, once fueron positivas para infección simple y una para infección múltiple (grupo de doce subtipos de alto riesgo: HPV-H y HPV-16).

De igual manera como lo reportan algunos estudios, se obtuvieron casos con reporte negativo para anormalidades de las células epiteliales en la clasificación de la citología, pero positivos para algunos subtipos HPV-H. ${ }^{2}$ Revisando el ciclo vital del HPV, la infección tiene dos fases: una latente y otra productiva. En la primera el DNA permanece en el núcleo en forma circular libre, sin producir atipia celular, sólo se puede detectar mediante método molecular. ${ }^{18,19}$

Diferente es la infección productiva, donde existe una actividad del DNA viral que permite ser detectado por la prueba molecular y además el cambio celular puede observarse tanto en la citología como en el estudio del tejido en la biopsia. ${ }^{20,21}$ Por diferentes circunstancias, a pesar de verse atipia celular en la citología, la prueba para biología molecular fue invalidada. ${ }^{22}$ Dentro de las causas reportadas tenemos que las muestras no siempre están sujetas a la temperatura adecuada, en especial durante el transporte. ${ }^{23}$

Una de las limitaciones de este estudio fue haber detectado solo la infección para el grupo de virus oncogénico, lo que no permitió explicar los casos con atipia celular del informe de citología con un reporte de DNA negativo. Una primera hipótesis podría ser que éste cambio se deba a la presencia de virus de bajo riesgo como HPV 6 y $11^{24}$, u otro subtipo. La segunda es que podría corresponder a un proceso o estadio regresivo, donde persista el cambio morfológico sin la presencia viral. ${ }^{25}$
Trabajar con subtipos de alto y bajo riesgo quizá permita establecer diagnósticos diferenciales ${ }^{26}$ con aquellos cambios celulares exuberantes que corresponden a procesos inflamatorios o bien a cambios regenerativos o reparativos frecuentes en las preparaciones de citología y que son motivo de interconsulta.

Este trabajo espera contribuir con una de las metas propuestas por el Ministerio de Salud y Protección Social en conjunto con el Instituto Nacional de Cáncer, en el Plan Nacional para el Control del Cáncer en Colombia 2012-2020, para la transición de tamización de cáncer de cuello uterino con citología a pruebas moleculares de detección temprana de HPV del año 2013. 27,28

Para proyección social de la Fundación Universitaria de Ciencias de la Salud y la Facultad de Citohistología es importante haber realizado esta primera campaña de citología en conjunto con la tipificación para HPV oncogénicos, permitiendo la aplicación de nuevas tecnologías y colaborando a disminuir el riesgo de mortalidad en nuestro país.

\section{Conclusión}

El uso de la PCR permitió identificar infecciones simples y múltiples por HPV de alto riesgo. Las mujeres con atipia celular (ASC-US y LSIL) fueron remitidas a colposcopia para su correspondiente estudio. No se había tenido experiencia en el uso de la citología en base líquida comparado con resultados de estudios virales moleculares (Cobas ${ }^{\circledR} 4800$ ). Así mismo fue un aprendizaje el enfoque multidisciplinario de las lesiones del cérvix, las cuales se abordaron desde la observación colposcópica, la realización de los dos tipos de citología y la intervención en el manejo de las pacientes.

\section{Referencias}

1. Dallenbach-Hellweg PH. Histopatología del cuello uterino: atlas a color. $1^{\mathrm{a}}$ ed. Madrid, España: Ediciones Journal; 2006.

2. Kjaer SK, van den Brule AJ, Paull G, Svare EI, Sherman ME, Thomsen BL, et al. Type specific persistence of high risk human papillomavirus (HPV) as indicator of high grade cervical squamous intraepithelial lesions in young women: population based prospective follow up study. BMJ. 2002 Sep 14; 325(7364):572. 
3. Walboomers JM, Jacobs MV, Manos MM, Bosch FX, Kummer JA, Shah KV, et al. Human papillomavirus is a necessary cause of invasive cervical cancer worldwide. J Pathol. 1999;189(1):12-9.

4. Grillo-Ardila CF, Martínez-Velásquez MY, Morales-López B. Virus del papiloma humano: aspectos moleculares y cáncer de cérvix. Rev Colomb Obstet Ginecol. 2008;59:310-5.

5. Ramzy I. Clinical cytopathology \& aspiration biopsy : fundamental principles and practice. Norwalk, Conn.: Appleton \& Lange; 1990.

6. zur Hausen H. Papillomaviruses and cancer: from basic studies to clinical application. Nat Rev Cancer. 2002;2(5):342-50.

7. Solomon D, Nayar R. El Sistema Bethesda para informar la citología cervical: definiciones, criterios y notas aclaratorias. Buenos Aires: Journal; 2005.

8. Lie AK, Risberg B, Borge B, Sandstad B, Delabie J, Rimala R, et al. DNA- versus RNA-based methods for human papillomavirus detection in cervical neoplasia. Gynecol Oncol. 2005 Jun;97(3):908-15.

9. Beldi MC, Tacla M, Caiaffa-Filho H, Ab'saber A, Siqueira S, Baracat EC, et al Implementing human papillomavirus testing in a public health hospital: challenges and opportunities. Acta Cytol.. 2012;56(2):160-5.

10. Wright TC Jr, Stoler MH, Sharma A, Zhang G, Behrens C, Wright TL. Evaluation of HPV-16 and HPV-18 genotyping for the triage of women with high-risk HPV+ cytology-negative results. Am J Clin Pathol. 2011 Oct; 136(4):578-86.

11. Saez de Santamaria J, Agustin Vazquez D. Cuadernos de Citopatologia: Citología líquida. Madrid : Díaz de Santos, c2006.

12. Campo Rodríguez P, Puerto de Amaya M. Comparación entre las técnicas de citologia compartida: convencional vs bases liquida. Repert med y cir. 2011;20:240-4.

13. De La Fuente-Villarreal D, Guzmán López S, Barboza-Quintana O, González Ramírez RA. Biologia del Virus del Papiloma Humano y tecnicas del diagnostico. Med Univer. 2010;12(49):231-8.

14. Cotran RS, Kumar V, Collins T, Robbins SL. Patología estructural y funcional [de] Robbins. Madrid, España: McGraw-Hill/Interamericana; 2000.

15. Wright TC Jr, Massad LS, Dunton CJ, Spitzer M, Wilkinson EJ, Solomon D. 2006 consensus guidelines for the management of women with cervical intraepithelial neoplasia or adenocarcinoma in situ. Am J Obstet Gynecol. 2007 Oct; 197(4):340-5.

16. Saslow D, Solomon D, Lawson HW, Killackey M, Kulasingam SL, Cain J, Garcia FA, et al. American Cancer Society, American Society for Colposcopy and Cervical Pathology, and American Society for Clinical Pathology screening gui- delines for the prevention and early detection of cervical cancer. CA Cancer J Clin. 2012 May-Jun; 62(3):147-72.

17. Spinillo A, Dal Bello B, Gardella B, Roccio M, Dacco MD, Silini EM. Multiple human papillomavirus infection and high grade cervical intraepithelial neoplasia among women with cytological diagnosis of atypical squamous cells of undetermined significance or low grade squamous intraepithelial lesions. Gynecol Oncol. 2009;113(1):115-9.

18. Bibbo M. Comprehensive cytopathology. Philadelphia: Saunders; 1997.

19. Wright T.C KR, Ferenczy A. Precancerous Lesions of the cervix. In: Kurman, Robert J., Hedrick Ellenson, Lora, Ronnett, Brigitte M, editors. Blaustein`s pathology of the female genital tract. New York: Springer; 2006. p. 253-324.

20. Manos MM, Kinney WK, Hurley LB, Sherman ME, Shieh-Ngai J, Kurman RJ, et al. Identifying women with cervical neoplasia: Using human papillomavirus dna testing for equivocal papanicolaou results. JAMA. 1999;281(17):1605-10.

21. Farag R, Redline R, Abdul-Karim FW. Value of combining HPV-DNA testing with follow-up Papanicolaou smear in patients with prior atypical squamous cells of undetermined significance. Acta cytol. 2008;52(3):294-6.

22. Dunne EF, Unger ER, Sternberg M, McQuillan G, Swan DC, Patel SS, et al. Prevalence of hpv infection among females in the united states. JAMA. 2007;297(8):813-9.

23. OMS. Control Integral del Cancer Cevicouterino: guía de practicas esenciales. Ginebra: OMS; 2011.

24. Jing Shu Y GE. comprehensive Cancer Cytopathology of the Cervix Uteri Correlation with histopathology. New York: McGraw-Hill;1995.

25. Munger K. The molecular biology of cervical cancer. J Cell Biochem Suppl. 1995;23: 55-60.

26. McKee GT. Cytopathology. London; Baltimore: Mosby-Wolfe; 1997.

27. Colombia. Ministerio de la Protección Social. Recomendaciones para la tamizacion de neoplasias de cuello uterino en mujeres sin antecedentes de patologia cervical (Preinvasora o Invasora) en Colombia: guia practica clinica numero 3 [monografía en Internet]. ]. Bogotá: Instituto Nacional de Cancerología; 2007 [citado 29 abr 2014]. Disponible en: http://www.cancer.gov.co/documentos/RecomendacionesyGuias/GuiaN3.pdf

28. Colombia. Ministerio de la Protección Social. Recomendaciones para la tamizacion de neoplasias de cuello uterino en mujeres sin antecedentes de patologia cervical (Preinvasora o Invasora) en Colombia: guia practica clinica numero 3 [monografía en Internet]. ]. Bogotá: Instituto Nacional de Cancerología; 2007 [citado 29 abr 2014]. Disponible en: http://www.cancer.gov.co/documentos/RecomendacionesyGuias/GuiaN3.pdf 